\title{
DIAGRAMS ASSOCIATED WITH SUBGROUPS OF FUCHSIAN GROUPS
}

\author{
by W. W. STOTHERS
}

(Received 10 June, 1977)

The use of graphs in the study of groups is well-established. In this paper, we wish to indicate how certain graph-like objects may be used in a similar way. A diagram is a pseudograph which may have some free edges, i.e. edges with just one end.

We discuss two kinds of diagram related to some Fuchsian groups. In the first section, we define D-diagrams for finitely generated Fuchsian groups of the first kind. From these, various kinds of information can be obtained. The technique is equivalent to the permutation description in [7] and [12]. Such diagrams were used in [11] for the modular group, and in [9] for a wider class of groups. In the second section, we define L-diagrams for the modular group, with a brief indication of the extension to other groups. Such diagrams were used in [6] for special subgroups of the modular group.

If $F$ is a finitely generated Fuchsian group of the first kind, then it has a presentation

$$
\begin{aligned}
F=\left\langle E_{1}, \ldots, E_{f}, P_{1}, \ldots, P_{h}, A_{1}, B_{1}, \ldots, A_{g}, B_{g}:\right. \\
\left.E_{1}^{n_{1}}=\ldots=E_{f}^{n_{f}}=\prod_{i=1}^{f} E_{i} \prod_{j=1}^{h} P_{j} \prod_{k=1}^{\mathrm{g}}\left[A_{k}, B_{k}\right]=I\right\rangle,
\end{aligned}
$$

where $n_{1}, \ldots, n_{f}$ are integers greater than one. Elements which are $F$-conjugate to a non-trivial power of some $E_{i}$ (resp. some $P_{j}$ ) are called elliptic (resp. parabolic). All other elements of $F-\{I\}$ are called hyperbolic. See [5].

1. D-diagrams. In this section, we do not allow free edges. Suppose that $D$ is a directed diagram in which each edge and loop is coloured by one of a finite set $C$ of colours. Then $D$ is well-coloured by $C$ if, for each $c \in C$, each vertex of $D$ has a $c$-loop or is the initial point of one $c$-edge and the end point of one $c$-edge.

If $D$ is well-coloured by $C$, then each $c \in C$ induces a permutation $\pi(c)$ of $V(D)$, the vertex set of $D$. For $c \in C$, we put $c^{-1}$ for a $c$-loop or $c$-edge of $D$ traversed in the opposite direction. Clearly, $(\pi(c))^{-1}=\pi\left(c^{-1}\right)$. Put $C_{1}=\left\{c^{-1}: c \in C\right\}$.

A walk (over $C$ ) is a finite sequence of elements of $C \cup C_{1}$. The walk $w=c_{1}, \ldots, c_{n}$ corresponds to the permutation $\pi(w)=\pi\left(c_{n}\right) \ldots \pi\left(c_{1}\right)$.

Suppose that $F$ has presentation (1). Let $C$ be a set of $f+h+2 g$ colours labelled by the generators of $F$. Then $D$ is a $D$-diagram for $F$ if

(a) $D$ is a directed diagram with a finite or countable vertex set and a distinguished vertex $P$,

(b) $D$ is well-coloured by $C$,

(c) for $i=1, \ldots, f, \pi\left(E_{i}^{n_{1}}\right)$ is the identity, identity,

(d) $\pi\left(E_{1}, \ldots, E_{f}, P_{1}, \ldots, P_{h}, A_{1}^{-1}, B_{1}^{-1}, A_{1}, B_{1}, \ldots, A_{\mathrm{g}}^{-1}, B_{\mathrm{g}}^{-1}, A_{\mathrm{g}}, B_{\mathrm{g}}\right)$ is the

(e) $D$ is connected.

Glasgow Math. J. 20 (1979) 103-114 
Condition (c) may be restated as

(c') for $i=1, \ldots, f$, the $E_{i}$-edges form $m$-gons, where $m \mid n_{i}$. An $E_{i}$-loop or an $E_{i}$-polygon with fewer than $n_{i}$ sides is a degenerate polygon.

Any element of $F$ can be expressed as a product of elements of $C \cup C_{1}$ and so determines a walk over $C$. The conditions on a D-diagram guarantee that two expressions for the same element of $F$ give the same permutation of the vertex set. Given a D-diagram $D$, it is clear that, if we use the same symbol for an element of $F$ and for a corresponding walk, then

$$
\begin{aligned}
G(D) & =\{w \in F: \pi(w) \text { fixes } P\} \\
& =\{w \in F: \text { edges of } w \text { determine a closed path at } P\}
\end{aligned}
$$

is a subgroup of $F$. Each right coset consists of elements which give a path from $P$ to a fixed vertex of $D$. Hence, the index of $G(D)$ in $F$ is equal to the number of vertices of $D$.

Conversely, a subgroup $G$ of $F$ determines $D(G)$, a D-diagram for $F$ in the following way. We begin with a set of vertices labelled by the right cosets of $G$ in $F$, with $G$ as the special vertex. For each $X \in C$, we add loops and edges of colour $X$ to indicate the effect of post-multiplication by $X$ on the cosets. The conditions (a) to (e) are clearly satisfied, (b) and (c) corresponding to the relations of $F$. Since $G X=G$ if and only if $X \in G$, $G(D(G))=G$. Also, if $D$ is a diagram for $F$, then $D(G(D))=D$, up to the labelling introduced on the former during the construction. We therefore have

THEOREM 1.1. There is a 1-1 correspondence between subgroups of index $n$ in $F$ and $D$-diagrams for $F$ with $n$ vertices.

The proof we have sketched is similar to that in [15] for the modular group. It is simpler than that in [1] in that it avoids Schreier systems. The generalization beyond the modular group is the graphical analogue of [8].

In itself, Theorem 1.1 is of use only for counting arguments, but we can also obtain geometric and algebraic information from D-diagrams.

Consider the elements of finite order in a subgroup $G$ of $F$. The elements of finite order in $F$ are precisely the elliptic elements. We observe that $X E_{i}^{m} X^{-1} \in G$ if and only if the walk $E_{i}^{m}$ has $\pi\left(E_{i}^{m}\right)(G X)=G X$. If $m$ is the least positive integer with $X E_{i}^{m} X^{-1} \in G$, then $m \mid n_{i}, G$ has an element of order $n_{i} / m$ and $D(G)$ has a simple closed $m$-gon of colour $E_{i}$ at vertex $G X$. The element is non-trivial if and only if the polygon at $G X$ is degenerate. Also, since $V E_{i}^{r}=E_{i}^{r} V$ with $V \in F, r \neq 0\left(\bmod n_{i}\right)$ implies that $V=E_{i}^{s}$, it is easy to see that $X E_{i}^{m} X^{-1}$ and $Y E_{i}^{n} Y^{-1}$ are $G$-conjugate if and only if $i=j, m=n$ and $G Y=G X E_{i}^{r}$ for some $r$. The last condition means that $G X$ and $G Y$ are on the same $E_{i}$-polygon in $D(G)$. Hence

THEOREM 1.2. If $G$ is a subgroup of $F$, the conjugacy classes of maximal finite subgroups of $G$ are in 1-1 correspondence with the degenerate polygons of $D(G)$.

Similarly, on considering the parabolic elements, we have

THEOREM 1.3. If $G$ is a subgroup of $F$, then the conjugacy classes of maximal parabolic subgroups of $G$ are in 1-1 correspondence with finite polygons of colour $P_{j}$ in $D(G)$. 
Corollary 1.4. If $D(G)$ has $h_{0}$ finite polygons in colours $P_{1}, \ldots, P_{h}$, then the fundamental domain for $G$ has $h_{0}$ inequivalent parabolic cusps.

It should be clear that the location of the cusps and elliptic vertices for a subgroup can be deduced from the D-diagram and knowledge of those for $F$. See Example 1 below.

The complete $\mathrm{D}$-diagrams are inconvenient from an algebraic point of view. Two different simplifications can be useful.

Consider first the case where $F$ has parabolic elements, i.e. $h>0$. We can omit the generator $P_{1}$ and the final relation from (1). Then $F$ is presented as a free product:

$$
F=C_{n_{1}} \times C_{n_{2}} \times \ldots \times C_{n_{4}} \times\left(C_{\infty}\right)^{n+2 g-1} .
$$

We write $C^{\prime}$ for the reduced set of colours. A $D_{1}$-diagram for $F$ is obtained from a $D$-diagram for $F$ by deleting the $P_{1}$-loops and edges. Then a $D_{1}$-diagram is a structure satisfying (a), (b), (c) and (e). Given a $\mathrm{D}_{1}$-diagram, we can recover a unique D-diagram by adding $P_{1}$-loops and edges to satisfy (d). There are obvious analogues of 1.1 and 1.2.

Suppose that $D$ is a $D_{1}$-diagram for $F$. A route is a walk $w$ over $C^{\prime}$ such that $\pi(w)$ fixes $P$ and which does not contain $c, c^{-1}$ as consecutive terms. Any walk $w$ for which $\pi(w)$ fixes $P$ determines a unique route (obtained by deleting instances of $c, c^{-1}$ as often as possible). Given routes $r_{1}, r_{2}$, the walk " $r_{1}$ then $r_{2}$ " gives a route which we call $r_{1} r_{2}$. With this operation, the routes of $D$ form a group $\mathscr{R}(D)$.

To determine the structure of $\mathscr{R}(D)$, we can adapt a standard graph theoretic technique. Given a $D_{1}$-diagram $D$, we take a spanning tree $T$ by deleting all loops and as many edges as necessary. Let $Q$ be any vertex of $D$. Then there is a sequence $t(Q)$ of edges of $T$ leading from $P$ to $Q$. As $T$ is a tree, $t(Q)$ is well-defined. If $Q_{1} Q_{2}$ is an edge of $D$ not in $T$, then it is a chord of $T$ and we associate with it the route $r\left(Q_{1} Q_{2}\right)=$ $t\left(Q_{1}\right), Q_{1} Q_{2}, t\left(Q_{2}\right)^{-1}$. If $e$ is a loop at $Q$, then there is a route $r(e)=t(Q), e, r(Q)^{-1}$.

THEOREM 1.5. If $D$ is a $D_{1}$-diagram and $T$ a spanning tree, then $\mathscr{R}(D)$ is the free group generated by the routes derived from the loops of $D$ and the chords of $T$.

The proof for a conventional graph is well known (see [2]); the extension requires no new ideas.

Some of the routes in a $D_{1}$-diagram come from the relations of $F$. For example, if $w$ is any walk, then $w E_{i}^{n} w^{-1}$ defines a route. Let $\mathscr{R}(F, D)$ denote the subgroup of $\mathscr{R}(D)$ generated by such routes. Clearly, $\mathscr{R}(F, D)$ is normal and we have

Theorem 1.6. If $D$ is a $D_{1}$-diagram, then $G(D)=\mathscr{R}(D) / \mathscr{R}(F, D)$.

CoROLLARY 1.7. (i) If $G$ is a subgroup of a finitely generated free group $F$, then $G$ is free.

(ii) If, further, $G$ has index $n<\infty$, then $G$ has rank $1+n(h+2 g-2)$.

Proof. The first part is obvious since, in this case, $\mathscr{R}(F, D(G))=\langle I\rangle$.

The second follows since $D(G)$ has $n(h+2 g-1)$ loops and edges, while a spanning tree requires exactly $n-1$ edges. 
This corollary is a special case of the classic theorem of Schreier. In fact, the complete theorem can be proved by relaxing the cardinality conditions in (1) and (a) above.

THEOREM 1.8. Let $F$ be a free product with expansion (2), and let $G$ be a subgroup of $F$. Then $G$ is a free product of finite cyclic groups (each with order dividing some $n_{i}$ ) and of infinite cyclic groups.

Proof. Let $D$ be the $D_{1}$-diagram for $G$, and let $T$ be a spanning tree for $D$. Suppose that $\mathscr{P}$ is an $m$-gon of colour $E_{i}$. As $T$ is a tree, $\mathscr{P}$ has at least one edge not in $T$. Suppose that the edges of $\mathscr{P}$ not in $T$ are, in order, $e_{1}, \ldots, e_{k}$. Then, if $e_{1}$ has initial point $Q$,

$$
r\left(e_{1}\right), r\left(e_{2}\right), \ldots, r\left(e_{k}\right)=t(Q), E_{i}^{m}, t(Q)^{-1} \text {. }
$$

Figure 1 gives an example, a formal proof is tedious. In the figure, the solid edges are edges belonging to $T$.

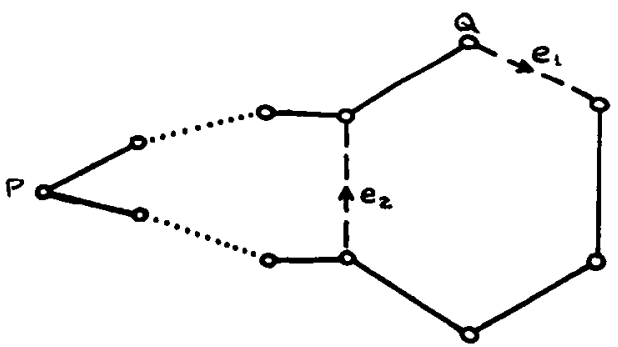

Figure 1

Since the $r\left(e_{\mathrm{i}}\right)$ are independent (by.1.5), we can obtain a new basis on replacing $r\left(e_{1}\right)$ by $t(Q), E_{i}^{m}, t(Q)^{-1}$. If the vertex $Q$ has an $E_{j}$-loop, then $r(Q)=t(Q), E_{j}, t(Q)^{-1}$. In the quotient $\mathscr{R}(D) / \mathscr{R}(F, D), t(Q), E_{i}^{m}, t(Q)^{-1}$ can be ignored if $m=n_{i}$, and is of order $n_{i} / m$ otherwise. Since all other generators are of infinite order and we have taken into account the generators of $\mathscr{R}(F, D)$, the result follows.

COROLlary 1.9. If, further, $G$ has finite index, then $G$ is a finite free product.

Proof. Since $D$ is finite, the set of generators of $\mathscr{R}(D)$ is finite.

EXAMPLE 1. We recall that the classical modular group $\Gamma$ has presentation

$$
\Gamma=\left\langle E_{1}, E_{2}, P_{1}: E_{1}^{2}=E_{2}^{3}=E_{1} E_{2} P_{1}=I\right\rangle .
$$

We realise $\Gamma$ as a group of bilinear transformations by taking

$$
E_{1}(z)=-1 / z ; E_{2}(z)=-1 /(z+1) ; P_{1}(z)=z-1 \text {. }
$$

The usual fundamental domain for $\Gamma$ has elliptic vertices of order 2 (resp. 3) at $i$ (resp. $\varphi=\frac{1}{2}(\sqrt{3} i-1)$ ), and a parabolic cusp at $i \infty$.

Figure 2 shows a $D_{1}$-diagram for $\Gamma$. Here, as later, $E_{1}$-edges occur in oppositely directed pairs. Such a pair is shown by a single undirected edge. 


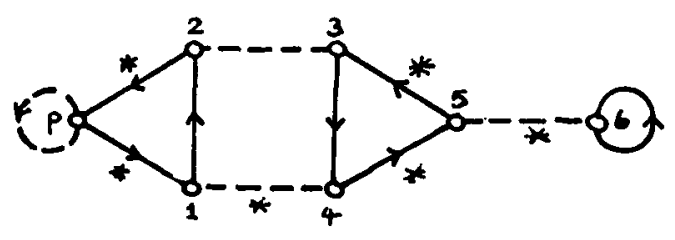

Figure 2

By Theorem 1.1, the corresponding subgroup $G$ has index 7. By Theorem 1.2, $G$ has one class of subgroups of order 2 , including $\left\langle E_{1}\right\rangle$, and one of subgroups of order 3 , including $\left\langle E_{3}=\left(E_{1} E_{2}\right)^{3} E_{2}\left(E_{1} E_{2}\right)^{-3}\right\rangle$. The corresponding $P_{1}$-edges are $(P, 3),(3,6),(6,5)$, $(5,1),(1, P),(2,4),(4,2)$. Using Theorem 1.3 , we have 2 inequivalent parabolic cusps, one at $i \infty$ fixed by $\left\langle P_{1}^{5}\right\rangle$ and one at $-1=E_{2}^{2}(i \infty)$ fixed by $\left\langle\left(E_{2}^{2} P_{1} E_{2}\right)^{2}\right\rangle$. Elliptic vertices occur at $i$ and at $\left(E_{1} E_{2}\right)^{3}(\varphi)$.

To describe the group $G$, we take the spanning tree consisting of the starred edges in Fig. 2. We obtain

$$
G=\left\langle E_{1}, E_{3}, E_{2} E_{1} E_{2}^{2} E_{1} E_{2}: E_{1}^{2}=E_{3}^{3}=I\right\rangle
$$

The third generator happens to be parabolic, being $E_{2}^{2} P_{1}^{2} E_{2}$. To obtain a presentation of type (1), some manipulation is needed.

An advantage of the $D_{1}$-diagrams is that they can be combined in a very simple way. Suppose that we have $n_{i}$ vertices with $E_{i}$-loops in a collection of $D_{1}$-diagrams, with at least one loop in each diagram. Then we can remove these loops and replace them with an $E_{i}$-coloured, directed $n_{i}$-gon. The result is a $\mathrm{D}_{1}$-diagram. Only one $P_{1}$-polygon is affected at each vertex, so the number of classes of elliptic and parabolic subgroups can be calculated from those in the original parts. This technique, known as joining, has been exploited in [11], where we have given an indication of the group theoretic interpretation.

Similar techniques can be used with D-diagrams for non-parabolic groups (i.e. groups with $h=0$ ). In such a case, the need to have condition (d) in the result causes some complication. One procedure, with many examples, is described in [10].

For internal consistency, we have described diagrams which differ slightly from those of [10], [11]. In those papers, we have taken the parabolic generator to be $E_{1} E_{2}$. Here, this is $P_{1}^{-1}$, so the green edges of those papers are the reverse of the $P_{1}$-edges in the present one. This does not affect the number or sizes of the polygons involved. Also, the pairs of $E_{1}$-edges correspond to the red edges of earlier papers.

In the case of a group with elliptic elements, i.e. with $f>0$, it may be useful to remove one of the $E_{i}$ from the generating set in (1). The diagram obtained by removing the $E_{i}$-loops and edges is a $D_{2}$-diagram. The conditions for a $D_{2}$-diagram are obtained by deleting from (c) the condition involving $E_{i}$, and by replacing (d) by

$$
\left(\pi\left(E_{i+1}, \ldots, E_{f}, P_{1}, \ldots, P_{h}, A_{1}^{-1}, \ldots, B_{g}, E_{1}, \ldots, E_{i-1}\right)\right)^{n_{t}}=1 .
$$

These diagrams have the advantage that the parabolic structure of the subgroup is clear. 
Analogues of 1.5 and 1.6 are straight-forward. With care, a nice generating set can be obtained, see [11]. We shall use $\mathrm{D}_{2}$-diagrams in the next section.

An obvious question about all these diagrams is the significance of the choice of special vertex. Suppose that $D$ is a $D$-diagram with a vertex $Q \neq P$. Let $w$ be an element of $F$ such that $\pi(w)(P)=Q$. Then a walk $v$ is closed at $Q$ if and only if $w v w^{-1}$ is closed at $P$, i.e. belongs to $G(D)$. Hence the group obtained from $D$ with $Q$ as distinguished vertex is conjugate to $G(D)$. An unlabelled $D$-diagram is a D-diagram with the label $P$ removed. We have

THEOREM 1.10. The conjugacy classes of subgroups of index $n$ in $F$ are in 1-1 correspondence with the unlabelled $D$-diagrams for $F$ with $n$ vertices.

It is clear that a subgroup is normal if and only if its D-diagram is not changed by moving the special vertex. This is equivalent to requiring that the automorphism group of the D-diagram is vertex-transitive. When the subgroup is normal, the D-diagram is a Cayley colour graph for the quotient group (see [13] for a discussion of such graphs). There does not seem to be an easy characterisation of the diagrams of normal subgroups.

The core $c(G)$ of a subgroup $G$ of the group $F$ is the largest normal subgroup of $F$ contained in $G$. Clearly, $c(G)=\cap_{x \in F} x^{-1} G x$. Since it is enough to take the intersection over coset representatives, $c(G)$ is the intersection of the subgroups obtained by varying the distinguished vertex in $D(G)$. With the observation before Theorem 1.1,

$$
c(G)=\{w \in F: \pi(w) \text { fixes all points of } D(G)\} .
$$

As a corollary, $F / c(G)$ is isomorphic to the group of permutations determined by the generators of $F$. Thus, $F / c(G)$ is determined by $D(G)$.

If we have groups $H \subseteq G \subseteq F$, with $F$ having presentation (1), then the diagrams corresponding to $H$ and to $G$ are related as follows. Each coset of $G$ in $F$ is the union of cosets $H$, so that $G$ induces a partition of the vertices of $D(H)$ (labelled as for Theorem 1.1). Suppose that $X$ is a generator of $F$. If $H Y, H Z$ belong to the same $G$-class $G Y$, then $H Y X$ and $H Z X$ belong to $G Y X$, so the edge structure in $D(H)$ is compatible with the partition. Thus, $D(G)$ is a "quotient diagram" of $D(H)$. A D-diagram is imprimitive if the vertex set has a partition compatible with the edge structure, otherwise it is primitive.

THEOREM 1.11. The maximal subgroups of $F$ are in 1-1 correspondence with the primitive $D$-diagrams for $F$.

Although we have described these diagrams in algebraic terms, there is also a geometric description. This may explain why both kinds of information can be obtained. We write $f(F)$ for a fundamental polygon of the Fuchsian group $F$. If $G$ is a subgroup of $F$, then we can take $f(G)$ as a simply connected union of $F$-translates of $f(F)$. With the identification of the sides of $f(G)$ indicated by the elements of $G$, we obtain a surface tesselated by copies of $f(F)$. Within each copy, we choose a point, adding edges and loops where copies meet along a side. Since the sides of $f(F)$ are paired by elements of $F$, we can direct and colour the loops and edges of the pseudograph obtained. Depending on the choice of $f(F)$, we get one of our $D_{i}$-diagrams drawn on the surface $f(G)$. 
As an example, we can take

$$
f(\Gamma)=\left\{z \in \mathbb{C}:|z| \geq 1 \text { and }-\frac{1}{2} \leq \operatorname{Re}(z) \leq \frac{1}{2}\right\} .
$$

The generator $P_{1}$ sends the side $\left\{z \in \mathbb{C}: \operatorname{Re}(z)=\frac{1}{2},|z| \geq 1\right\}$ to the side $\left\{z \in \mathbb{C}: \operatorname{Re}(z)=-\frac{1}{2}\right.$, $|z| \geq 1\}$. The generator $E_{1}$ interchanges the sides $\{z \in \mathbb{C}:|z|=1, \operatorname{Re}(z) \geq 0\},\{z \in \mathbb{C}:|z|=1$, $\operatorname{Re}(z) \leq 0\}$. Using this fundamental polygon, we obtain the $\mathrm{D}_{2}$-diagram for the subgroup.

Figure 3 shows a fundamental domain for the subgroup whose diagram is shown in Fig. 2. The lower part shows the derived $D_{2}$-diagram. The broken edges correspond to $E_{1}$, the solid edges to $P_{1}$.

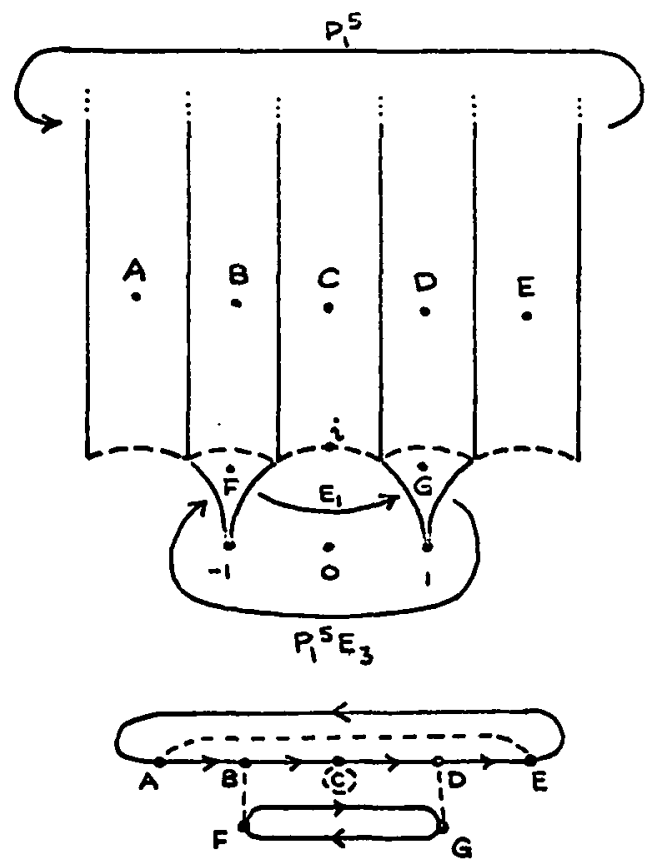

Figure 3

Besides providing an alternative approach to diagrams, this also shows that, when the genus of $f(G)$ can be defined, the $\mathrm{D}_{2}$-diagram can be drawn without intersection on a surface of this genus. Thus, the subgroups of genus zero in the modular group are precisely those with planar diagrams.

In certain circumstances, a pseudograph may be interpreted as a diagram for more than one Fuchsian group. Suppose that $F$ has the presentation (1), and that $F^{*}$ has a presentation of similar type with generators and parameters distinguished by asterisks. Suppose, further, that

(i) $f+h=f^{*}+h^{*}$,

(ii) $f \geq f^{*}$,

(iii) $n_{i} \mid n_{i}^{*}, i=1, \ldots, f^{*}$. 
Then a diagram for $F$ is (up to renaming of colours) a diagram for $F^{*}$ since the conditions on the former clearly include those on the latter. This is a diagramatic interpretation of the fact that $F=F^{*} / N$, where $N$ is the normal closure in $F^{*}$ of the set

$$
\mathcal{N}=\left\{\left(E_{i}^{*}\right)^{n_{i}}: i=1, \ldots, f^{*}\right\} \cup\left\{\left(P_{i}^{*}\right)^{n_{i}}: j=f^{*}+1, \ldots, f\right\} .
$$

EXAMPLE 2. Let $\Delta$ be the $(2,3,7)$-triangle group, i.e.

$$
\Delta=\left\langle E_{1}^{*}, E_{2}^{*}, E_{3}^{*}: E_{1}^{*^{2}}=E_{2}^{* 3}=E_{3}^{* \gamma}=E_{1}^{*} E_{2}^{*} E_{3}^{*}=I\right\rangle .
$$

Then we can take $F=\Delta, F^{*}=\Gamma$, so that (i), (ii) and (iii) are satisfied.

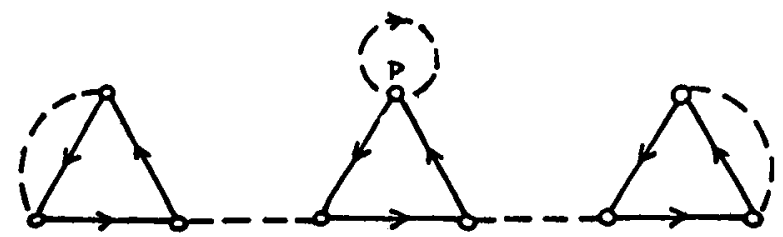

Figure 4

Figure 4 shows a $D_{2}$-diagram for $\Delta$. The corresponding subgroup $G_{1}$ has index 9 in $\Delta$ and is a congruence subgroup (corresponding to the prime (2) in the real subfield of $\mathbb{Q}(\exp (\pi i / 7)))$. The figure also represents a subgroup $G_{2}$ of index 9 in $\Gamma$. Since there are three parabolic classes, of width 1,1 and $7, G_{2}$ has level 7 . By Wohlfahrt's Theorem, a congruence subgroup of level 7 in $\Gamma$ must have index dividing 168 , so that $G_{2}$ is non-congruence (see [14]). This example shows that it is not possible to give a general rule for identifying diagrams belonging to congruence subgroups.

Since $\Delta / c\left(G_{1}\right)$ and $\Gamma / c\left(G_{2}\right)$ can be obtained from the diagram, they are isomorphic. It is not difficult to see that Fig. 4 shows the only unlabelled $D_{2}$-diagram for $\Delta$ with 9 vertices. Since PSL $\left(2,2^{3}\right)$ is a $(2,3,7)$ group with a subgroup of index 9 , this must be the factor group.

In this case, it is quite easy to see that the subgroups of $\Gamma$ which come from diagrams for $\Delta$ are precisely those of level 7 (see [10]).

2. L-diagrams. We consider the modular group with presentation (3). As in [11], we associate with a subgroup $G$ of $\Gamma$ a list of non-negative integers $\left(r, s, t_{1}, h_{0}, h_{\infty}\right)$, the short specification of $G$. By Theorem 1.2, $r$ (resp. $s$ ) is equal to the number of $E_{1}$ (resp. $E_{2}$ ) loops in $D(G)$. The group $G$ acts in the obvious way on $\mathbb{Q}^{*}=\mathbb{Q} \cup\{i \infty\}$. The elements of $\mathbb{Q}^{*}$ fall into $h=h_{0}+h_{\infty}$ classes. Within each class, the stabilizers are $G$-conjugate. By Theorem 1.3, $h_{0}$ is the number of classes with non-trivial stabilizer. Each such stabilizer is generated by a $\Gamma$-conjugate of $P_{1}^{c}$ for a positive integer $c$. This $c$ is the cusp-width of the relevant class. The sequence of cusp-widths is the cusp-split of $G,\left(c(1), \ldots, c\left(h_{0}\right)\right)$. The specification of $G$ is the list $\left(r, s, t_{1}, h_{0}, h_{\infty}, c(1), \ldots, c\left(h_{0}\right)\right)$. For $j=1$, we put $h(j)=$ $\left\{k: 1 \leq k \leq h_{0}, c(k)=j\right\}$, so that $D(G)$ has $h(1) P_{1}$-loops, $h(2) P_{1}$-coloured 2-gons, etc..

A $K$-diagram is a finite or countable diagram such that the edges incident with the vertex $A$ are labelled by $\mathbb{Z}(\bmod n)$, where $n$ is the degree of $A$. We allow $n=\infty$. The 
labelling of multiple edges is straight-forward, while a loop receives 2 labels and so contributes 2 to the degree of the vertex. A free edge has just one label. In many cases, it is possible to draw the $\mathrm{K}$-diagram with the edges ordered clockwise at each vertex. This avoids the need for explicit labelling.

Suppose that $K$ is a $\mathrm{K}$-diagram, and that $A$ is a vertex of $K$. For an integer $a$, the edge $a+1$ at $A$ is the successor of edge $a$ at $A$. Then $K$ has the triangle property if, when $A C$ is the successor of $A B$ at $A$, then there is an edge $B C$ such that $B C$ is the successor of $A C$ at $C$ and $A B$ the successor of $B C$ at $B$. Note that $A, B$ and $C$ need not be distinct since a loop or free edge may be involved. Also, the edges $A B, B C, A C$ need not be distinct. Figure 5 shows three cases of degeneracy.
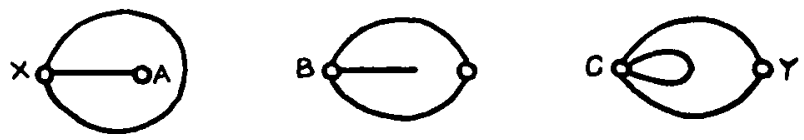

Figure 5

In each case, only the relevant edges and vertices are shown. In the first case, $A$ has degree 1 and $X A$ contributes 2 edges to a single "triangle". In the second case, there is a free edge at $B$. This is in just one triangle. In the third case, the loop at $C$ is one side of triangle $C Y C$, and itself constitutes a degenerate triangle at $C$.

An $L$-diagram is a $\mathrm{K}$-diagram with the triangle property. We note that, if an L-diagram has no degenerate triangles or loops and can be drawn without intersections and properly ordered incidences on a plane, then we have a triangulation of the plane.

Suppose that $G$ is a subgroup of $\Gamma$ and that $D=D(G)$. Using $D$, we can construct an L-diagram $L$. Each vertex of $L$ corresponds to a $P_{1}$ polygon (perhaps infinite) in $D$. Two vertices in $L$ are joined if there is a pair of $E_{1}$ edges in $D$ joining vertices in the corresponding polygons. The labels are determined by the orientation of the $P_{1}$ polygons. An $E_{1}$ loop gives rise to a free edge. Figure 6 shows the L-diagram corresponding to the diagram in Fig. 4.

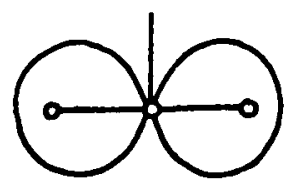

Figure 6

Given an L-diagram $L$, we can recover a $\mathrm{D}_{2}$-(and hence a $\mathrm{D}_{\text {-) }}$ diagram. Round each vertex of $L$ we draw a small circle meeting only the loops and edges from that vertex. Each intersection is a vertex of a new diagram $D$. Round each of the circles, the vertices are joined to give a polygon which is coloured $P_{1}$ and directed according to the labelling of the edges and loops in $L$. The portions of loops and edges outside the circles give pairs of edges which are coloured $E_{1}$. Free edges give $E_{1}$-loops. The triangle property in $L$ 
guarantees that $D$ is a $D_{2}$-diagram, i.e. we can add $E_{2}$ loops and edges to get a $D$-diagram for $\Gamma$, at least up to the choice of a special vertex in $D$. From earlier results we have

THEOREM 2.1. (i) There is a 1-1 correspondence between conjugacy classes of subgroups of $\Gamma$ and $L$-diagrams.

(ii) If $G$ is a subgroup of $\Gamma$ and $L$ a corresponding $L$-diagram, then

(a) $r$ is the number of free edges in $L$,

(b) $s$ is the number of degenerate triangles in $L$,

(c) $h$ is the number of vertices of $L$ and, for each positive integer $j, h(j)$ is the number of vertices of degree $j$. Thus $h_{0}$ is the number of vertices of finite degree.

The analysis of L-diagrams is complicated by the possibility of loops and free edges. In a later paper, we hope to discuss some of the results which can be obtained. At present, we concentrate on the construction of L-diagrams to give two existence results which extend the main theorem in [11].

THEOREM 2.2. If $\mathscr{I}=(c(i): i=1,2, \ldots)$ is a sequence of integers each at least 6 , then there is a subgroup of $\Gamma$ with $r=s=h_{\infty}=0$ and with $\mathscr{I}$ as its cusp-split.

Proof. By 2.1, we have to construct an L-diagram without free edges and degenerate triangles. Since each such feature implies the existence of a loop (see Fig. 5), an L-diagram without loops has $r=s=0$. To get $h_{\infty}=0$ and cusp-split $\mathscr{I}$, we must ensure that the vertices have degrees $c(1), c(2), \ldots$.

The L-diagram is drawn in stages. Let $C(k), k=0,1, \ldots$, denote the circle with centre $P$ and radius $k$. For $j \geq 1$, we construct a pseudograph $S(j)$ lying in and on $C(j)$. For $j \geq 2, S(j)$ is a subgraph of $S(j+1)$.

The vertex set of $S(1)$ consists of $P$ and $c(1)$ points on $C(1)$. The edge set consists of the arcs of $C(1)$ and radii joining $P$ to each of the points on $C(1)$. We may as well take $S(1)$ symmetrical.

Suppose that we have constructed $S(j)$ and that

(a) the vertices inside $C(j)$ have degrees $c(1), \ldots, c\left(n_{j}\right)$,

(b) the vertices on $C(j)$ are numbered $n_{j}+1, \ldots, n_{j}+m_{j}$ (clockwise), with vertex $k$ having degree $a(k)=3$ or 4 ,

(c) the edges within $C(j)$ each belong to two triangles,

(d) the edges on $C(j)$ each belong to one triangle, and their union is the whole of $C(j)$.

If $X$ and $Y$ are adjacent vertices on $C(j)$, we add a vertex $v\{X, Y\}$ on the arc of $C(j+1)$ within the sector bounded by $P X$ and $P Y$. If, further, $Y$ is vertex $k$ on $C(j)$ and lies between $X$ and $Z$, then we join $Y$ to $v\{X, Y\}$, to $v\{Y, Z\}$, and to $c(k)-a(k)-2(\geq 0$ by (b)) points on the arc $v\{X, Y\}, v\{Y, Z\}$. In each case, we choose the smaller of the possible arcs. The other edges of $S(j+1)$ are the arcs of $C(j+1)$ joining adjacent vertices. It should be clear that the $v\{X, Y\}$ have degree 4 while the other vertices on $C(j+1)$ have degree 3 , so $S(j+1)$ can be numbered as in (b). From the construction, $S(j+1)$ also satisfies (a), (c) and (d), so that the construction can proceed. 
In the obvious sense, the limit of the $S(j)$ is an L-diagram. It is clear that it is of the required type since, after the $j$ th stage, the degrees of vertices within $C(j)$ are not changed.

THEOREM 2.3. If $\mathscr{I}$ is as in 2.2 , then there is a subgroup of $\Gamma$ with $r=s=0, h_{\infty}=1$ and with cusp-split $\mathscr{}$.

Proof. As in 2.2, we construct an L-diagram in stages, but here we add an infinite set of vertices at each stage.

We take the origin of the coordinate plane as the vertex $P$. Let $D(j)$ be the line $y=j$ $(j \geq 1)$. The $D(j)$ replace the $C(j)$ of 2.2 .

The vertex set of $S(1)$ consists of $P$ and the points $\{(i, 1): i \in \mathbb{Z}\}$ on $D(1)$. The edge set of $S(1)$ consists of the segments of $D(1)$ together with edges joining $P$ to each vertex on $D(1)$. The vertex $(i, 1)$ is labelled $2 i+1$ and has degree 3 (in $S(1)$ ).

Now consider $j \geq 1$. Suppose that $X(x, j), Y(y, j)$ and $Z(z, j)$ are vertices of $S(j)$ with $Y$ adjacent to $X$ and to $Z$, that $Y$ is labelled by (the integer) $k$ and has degree $a(k)$. We add new vertices $A\left(\frac{1}{2}(x+y), j+1\right), B\left(\frac{1}{2}(y+z), j+1\right)$ and edges $A X, A Y, B X, B Y$. On the segment $A B$ of $D(j+1)$, we add $c(k)-a(k)-2$ further vertices, each joined to $Y$. Then $Y$ has degree $c(k)$ in $S(j+1)$. We repeat this process for each such triple of vertices of $S(j)$. We add edges between each adjacent pair of vertices on $D(j+1)$ to complete the construction of $S(j+1)$. All the new vertices have degree 3 or 4 in $S(j+1)$, so that the construction can continue. The added vertices are labelled by the integers $\left\{2^{j}(2 i+1): i \in\right.$ $\mathbb{Z}\}$.

Once again, the "limit" of the $S(j)$ is an L-diagram and gives a cusp-split of the required type.

Minor alterations in the above proofs would allow us to consider sequences with some terms equal to 5 . More detailed considerations lead to more general existence theorems. Observe that the construction in 2.2 is a generalisation of that in [6].

The idea behind L-diagrams extends to groups of type $(2, m, \infty)$, i.e. to the groups $F(m, \infty)$ with presentations

$$
F(m, \infty)=\left\langle X, Y: X^{2}=Y^{m}=I\right\rangle .
$$

Suppose that $K$ is a $K$-diagram and that $A B$ is edge $b$ at $B$ and the successor of edge $a$ at $A$. Then $(B, b)$ is the first successor of $(A, a)$. For an integer $n \geq 2$, the $n$th successor of $(A, a)$ is the $(n-1)$ th successor of the first successor. A K-diagram has the $m$-gon property if, for each vertex-integer pair $(A, a)$, the $m$ th successor of $(A, a)$ is $(A, a)$. This means that the successive edges at the vertices form (possibly degenerate) $m$-gons.

Exactly as for 2.3 , we have

THEOREM 2.4. There is a 1-1 correspondence between $K$-diagrams with the $m$-gon property and conjugacy classes of subgroups of $F(m, \infty)$.

The degrees of the vertices of such a diagram correspond to the "cusp-split" of the associated subgroup, i.e. to the "cycle pattern" of $X Y$. 
We can further extend the notion to the groups

$$
F(m, n)=\left\langle X, Y: X^{2}=Y^{m}=(X Y)^{n}=I\right\rangle .
$$

The correspondence is now with $\mathrm{K}$-diagrams which have

(i) the $m$-gon property, and

(ii) the degree of each vertex divides $n$.

These restrictions are so strong that it is doubtful whether the diagrams are of much practical value. Some results have been obtained for $F(3,7)$ by $L$. A. Best using (essentially) K-diagrams. Best's results are unpublished, but include a review of all subgroups of index $\leq 42$.

PostSCRIPT. The methods of the first section have been used for more general groups, see [3], [4]. These contain proofs of the full Kurosh Subgroup Theorem. The restriction to our Fuchsian groups leads to the relatively simple proof of 1.8 . The second of these papers has many interesting applications of the method.

\section{REFERENCES} 61-79.

1. I. M. S. Dey, Schreier systems in free products, Proc. Glasgow Math. Assoc. 7 (1965),

2. F. Hararay, Graph theory (Addison-Wesley, 1969).

3. P. J. Higgins, Notes on categories and groupoids, Mathematical Studies 32 (Van Nostrand Reinhold, 1971).

4. W. Imrich, Subgroup theorems and graphs, Lecture Notes in Mathematics 622 (SpringerVerlag, 1977).

5. J. Lehner, Discontinuous groups and automorphic functions (Amer. Math. Soc. 1964).

6. R. Lyndon, Two notes on Rankin's book on the modular group, J. Austral. Math. Soc. 16 (1973), 454-457.

7. M. H. Millington, Subgroups of the classical modular group, J. London Math. Soc. (2) 1 (1970), 351-357.

8. D. Singerman, Subgroups of Fuchsian groups and finite permutation groups, Bull. London Math. Soc. 2 (1970), 319-323.

9. W. W. Stothers, Free subgroups of the free product of cyclic groups, Math. Comput. 32 (1978), 1274-1280.

10. W. W. Stothers, Subgroups of the (2,3,7)-triangle group, Manuscripta Math. 20 (1977), 323-334.

11. W. W. Stothers, Subgroups of infinite index in the modular group, Glasgow Math. J. 19 (1978), 33-43.

12. C. Tretkoff, Non-parabolic subgroups of the modular group, Glasgow Math. J. 16 (1975), 91-102.

13. A. T. White, Graphs, groups and surfaces (North-Holland, 1973).

14. K. Wohlfahrt, An extension of F. Klein's level concept, Mlinois J. Math. 8 (1964), 529-535.

15. K. Wohlfahrt, Über einen Satz von Dey und die Modulgruppe, Arch. Math. (Basel) 29 (1977), 455-457.

\section{UNIVERSITY OF GLASGOW \\ GLASGOW \\ G12 8QW}

